'Sección de Reumatología del Hospital General de Agudos

"Dr. I. Pirovano". Buenos Aires, Argentina.

${ }^{2}$ Servicio de Reumatología del Hospital Británico de Buenos Aires.

${ }^{3}$ Unidad de Laboratorio Central del Hospital General de Agudos "Dr. I. Pirovano". Buenos Aires, Argentina.

${ }^{4}$ Sección de Reumatología del Instituto de Rehabilitación

Psicofísica (IREP). Buenos Aires, Argentina.

Recibido el 21 de septiembre de 2013, aceptado el 28 de octubre de 2014

Correspondencia a: Dr. Rodríguez GR Monroe 3550

Código postal 1430 Ciudad Autónoma de Buenos Aires, Argentina. Fax: +54-011-4825 7310 grrodrigue@intramed.net

\section{Frecuencia de anticuerpos para diagnóstico de enfermedad celíaca en pacientes con enfermedades del tejido conectivo y artropatías inflamatorias}

\author{
GRACIELA RITA RODRÍGUEZ ${ }^{1}$, FEDERICO ZAZZETTI², \\ SILVIA RAQUEL DA REPRESENTAÇAO ${ }^{3}$, MARÍA VERÓNICA LENCINA ${ }^{4}$, \\ JUAN CARLOS BARREIRA², KARINA ELIZABETH ÁLVAREZ ${ }^{1}$ \\ Serum anti endomysial and anti \\ transglutaminase antibodies in patients \\ with connective tissue diseases
}

Background: The detection of anti-transglutaminase IgA (tTG) and antiendomysial (EMA) is used for screening of celiac disease (CD) with a sensitivity and specificity of 90 and $99 \%$ respectively. There is an association between $C D$ and connective tissue diseases (CTD). Aim: To report the frequency of IgA $t T G$ and EMA in patients with a definite diagnosis of CTD and inflammatory arthropathies (IA). Material and Methods: One hundred forty nine patients, aged 19 to 86 years (133 females) with CTD and IA were studied. $t T G$ were determined by ELISA and EMA by indirect immunofluorescence. Results: Eight participants had at least one positive antibody (5.4\%, confidence intervals $(C I)=1.8-9)$, six had both (4.0\% CI=0.9-7.2) and two had only $t T G$ positive. An intestinal biopsy was performed in four of these participants, finding a marked villous atrophy in three and partial atrophy in one. Conclusions: Five percent of this group of patients with CTD or IA had positive antibodies for CD.

(Rev Med Chile 2014; 142: 1510-1516)

Key words: Autoimmunity; Celiac disease; Connective Tissue Diseases; Rheumatic Diseases.
L a enfermedad celíaca (EC) es una enteropatía autoinmune desencadenada por la ingesta de gliadina, proteína contenida en el trigo y otros cereales como el centeno y la cebada, en individuos genéticamente predispuestos. Esta intolerancia provoca un daño intestinal de distinta magnitud que revierte con la supresión del gluten de la dieta. En los últimos años, la aparición de nuevas y más exactas pruebas serológicas, además del reconocimiento de las distintas formas de expresión clínica de la enfermedad, han permitido conocer que es una afección relativamente frecuente afectando a 1 de cada 120-130 individuos en Estados Unidos de Norteamérica y Europa ${ }^{1}$.
En Argentina, un estudio en población general demostró una prevalencia de 1/167 individuos ${ }^{2}$.

Aunque se desconoce la causa, en las últimas décadas, el porcentaje de pacientes que se presentan con los síntomas clásicos (diarrea crónica, síndrome de malabsorción) ha disminuido; observándose una mayor proporción de formas clínicas atípicas o subclínicas ${ }^{3}$. Mientras que la biopsia de intestino delgado continúa siendo el gold standard para el diagnóstico de EC, en este grupo de pacientes pauci o asintomáticos, las pruebas serológicas son importantes para determinar aquellos pacientes que deberán ser referidos a biopsia. Las pautas propuestas por la Conferencia de Consenso 
del Instituto Nacional de la Salud (NIH) en junio de 2004, recomendaron la utilización del título de IgA antitransglutaminasa (tTG) o antiendomisio (EMA) para la investigación de EC junto al título de IgA sérica total; basándose en una sensibilidad de $90 \%$ y una especificidad de $99 \%$. Sin embargo, en individuos con deficiencia de IgA, que puede observarse hasta en $2 \%$ asociada a $\mathrm{EC}^{4}$, estas pruebas pueden resultar en falsos negativos, recomendándose en dichos casos la detección de IgG-tTG o IgG-EMA ${ }^{5}$.

La asociación entre EC y enfermedades del tejido conectivo (ETC) ha sido comunicada en por lo menos 13 enfermedades de fisiopatogenia autoinmune, particularmente con referencia a síndrome de Sjögren (SS) y artritis psoriásica ${ }^{6-9}$. Los pacientes con SS, por ejemplo, tienen una prevalencia de EC de alrededor de $15 \%$, recomendándose en la actualidad el screening serológico en este grupo de alto riesgo ${ }^{6,7,9}$. Asimismo, se ha descrito una artritis seronegativa como manifestación extraintestinal de la EC hasta en $26 \%$ de los pacientes celíacos comparado con 7,5\% de los controles ${ }^{10}$. La artritis reumatoidea (AR) también ha sido asociada a la $\mathrm{EC}^{1}$; apoyando esta observación la detección de anti-tTG en suero y líquido sinovial de pacientes con AR y otras artropatías ${ }^{11}$.

En base a lo expuesto anteriormente, podemos afirmar que existe una asociación comprobada entre la EC y distintas ETC y artropatías inflamatorias (AI); sin embargo, en nuestro conocimiento, no existen publicaciones en Argentina que aborden esta asociación. Por lo tanto, el objetivo de este estudio fue describir la frecuencia de positividad para IgA tTG y EMA en pacientes con diagnóstico definido de ETC y AI.

\section{Materiales y Método}

Se llevó a cabo un estudio descriptivo de corte transversal. Se incluyeron pacientes ambulatorios consecutivos $\geq$ de 18 años con diagnóstico de ETC definida según criterios de clasificación del American College of Rheumatology (ACR) incluyendo: $\mathrm{AR}, \mathrm{SS}$, lupus eritematoso sistémico (LES), artritis psoriásica (APs), artritis reactiva (ARe), esclerosis sistémica difusa o limitada (ESd o ESl), polimiositis $(\mathrm{PM})$, dermatomiositis $(\mathrm{DM})$, enfermedad mixta del tejido conectivo (EMTC), enfermedad indiferenciada del tejido conectivo (EITC) y AI indiferenciadas evaluados entre mayo de 2008 y junio de 2011 en consultorio externo por los Servicios de Reumatología de los centros de derivación participantes. Se destinó un consultorio de $2 \mathrm{~h}$ semanales al que concurrían pacientes referidos de los 3 centros intervinientes con diversas patologías reumatológicas. Luego, aquellos con diagnóstico de enfermedades autoinmunes sistémicas eran derivados al laboratorio para la realización de los anticuerpos.

Se registraron datos de variables demográficas (edad, sexo) y datos clínicos, incluyendo diagnóstico principal y diagnósticos secundarios, fecha de diagnóstico de la ETC o AI, manifestaciones articulares y extraarticulares, antecedentes familiares de ETC y enfermedades autoinmunes y síntomas gastrointestinales. El examen físico incluyó piel, aparato locomotor y semiología visceral. Se excluyeron pacientes con diagnóstico previamente conocido de EC.

Entre las pruebas de laboratorio solicitadas periódicamente a estos pacientes como control de su enfermedad de base, se incluyó la detección de anticuerpos para EC según un algoritmo diagnóstico preestablecido (Figura 1). Los pacientes con hallazgo de anticuerpos positivos fueron referidos a los Servicios de Gastroenterología de las instituciones intervinientes para la realización de la biopsia de intestino delgado. Para la detección de anti-tTG se empleó el método de ensayo por inmunoabsorción ligado a enzimas (ELISA) utilizando kits comerciales (INOVA Diagnostics, San Diego, CA). Para la detección de EMA se utilizó el método de inmunofluorescencia indirecta (IFI) mediante un kit comercial (BioSystems S.A, Barcelona) que utiliza como sustrato esófago de mono. Los kits utilizados para determinar ambos anticuerpos fueron de tipo IgA. Se consideraron positivos valores $\geq \mathrm{a} 20$ para anti-tTG y para EMA, se realizaron diluciones de 1:5, considerándose positivas a aquellas iguales o mayores a esta dilución, según las recomendaciones del fabricante. Se utilizaron los mismos kits comerciales durante todo el estudio.

El registro de datos siguió los procedimientos recomendados por el Comité de Revisión Institucional de los centros intervinientes. Durante todo el proceso de recolección de datos se siguieron los principios establecidos por la declaración de Helsinki. 


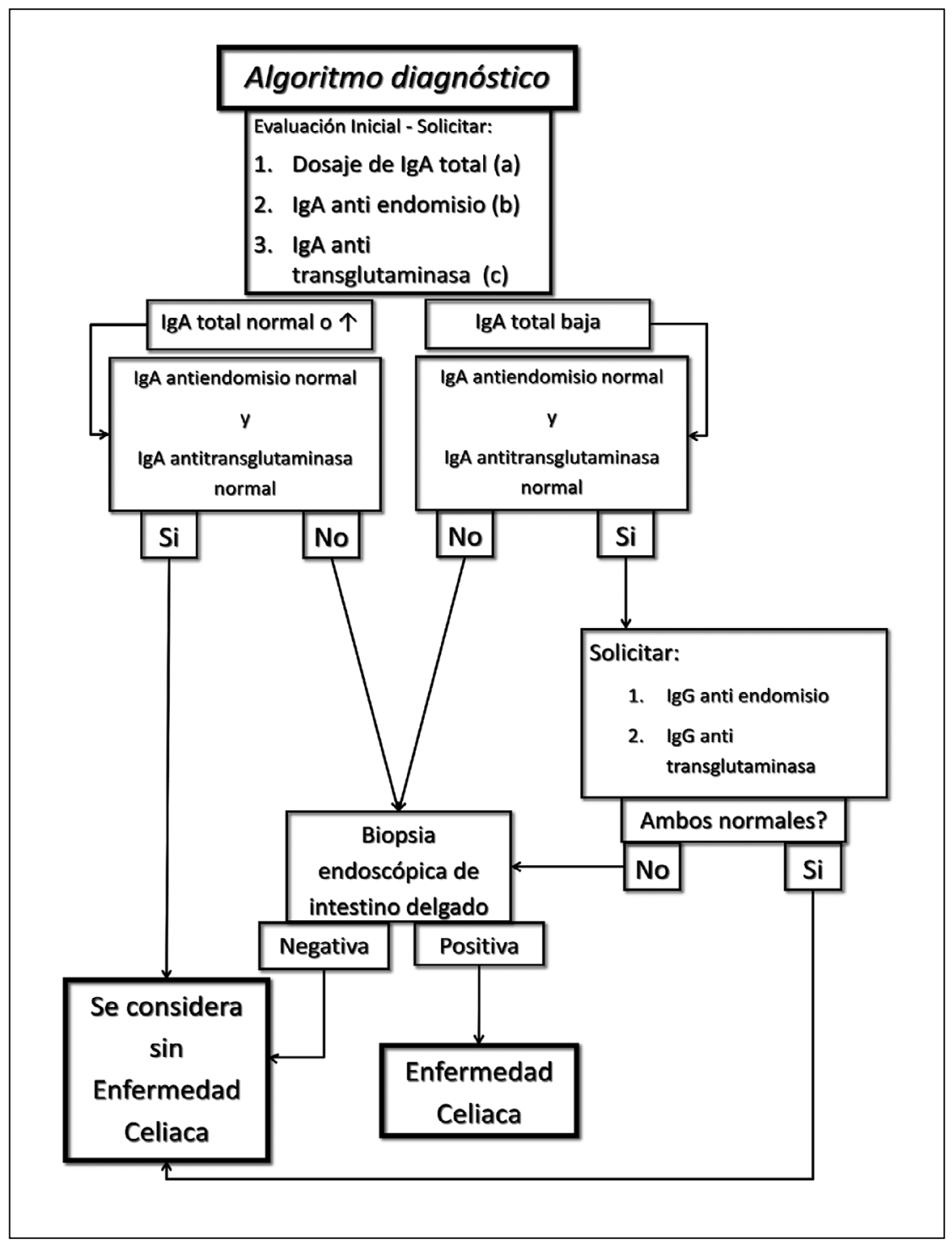

Figura 1. Algoritmo diagnóstico de enfermedad celíaca propuesto por el NIH en 2004. (a) Nefelometría; (b) Inmunofluorescencia Indirecta (IFI), substrato tercio inferior de esófago de mono. Biolinker, Barcelona; (c) Inmunoensayo enzimático de micropartículas (MEIA). Inova, San Diego.

\section{Análisis estadístico}

Los resultados fueron informados como medianas para variables numéricas y porcentajes para categóricas. La edad se informó como promedio \pm desvío estándar. Los intervalos de confianza (IC) fueron calculados para un valor de 95\%. Se utilizó test de Student para comparar variables continuas. Se tomó un valor de $\mathrm{p}<0,05$ para indicar significancia estadística. El análisis estadístico se realizó mediante el programa Intercooled STATA 10.0.

\section{Resultados}

Se incluyeron 149 pacientes (todos con IgA normal) en quienes se analizaron los datos de anticuerpos en 76 AR, 26 LES, 12 SS, 7 ES, 7 APs, 6 miopatías inflamatorias (PM/DM), 6 AI, 4 EITC, 4 ARe y 1 EMTC. En la Tabla 1 se presentan las características demográficas de los pacientes analizados.

De los 149 , ocho pacientes $(5,37 \%$; IC $=1,75$ $8,99)$ presentaron al menos un anticuerpo positivo 
Anticuerpos para enfermedad celíaca en enfermedades reumatológicas - G. R. Rodríguez et al

Tabla 1. Características demográficas de 149 pacientes con enfermedades del tejido conectivo y artropatías inflamatorias

\begin{tabular}{|c|c|}
\hline Total pacientes & $\begin{array}{c}149 \\
\text { n (\%) }\end{array}$ \\
\hline Edad (años)* & $44,79(19-86)$ \\
\hline Sexo (femenino) & $133(89,26)$ \\
\hline Tiempo de evolución (meses) & 64,1 \\
\hline \multicolumn{2}{|l|}{ ETC } \\
\hline Artritis reumatoidea & $76(51,01)$ \\
\hline Lupus eritematoso sistémico & $26(17,45)$ \\
\hline Síndrome de Sjögren & $12(8,05)$ \\
\hline Esclerosis sistémica & $7 \quad(4,70)$ \\
\hline Artritis psoriásica & $7 \quad(4,70)$ \\
\hline Miopatía inflamatoria idiopática & $6(4,03)$ \\
\hline Artritis indiferenciada & $6(4,03)$ \\
\hline EITC & $4(2,68)$ \\
\hline Artritis reactiva & $4(2,68)$ \\
\hline EMTC & $1 \quad(0,67)$ \\
\hline \multicolumn{2}{|l|}{ Anticuerpos positivos } \\
\hline Anti-tTG & $8 \quad(5,37)$ \\
\hline EMA & $6(4,03)$ \\
\hline Diagnóstico de EC & $4(2,68)$ \\
\hline
\end{tabular}

${ }^{*}$ Media (rango). ETC = Enfermedad del tejido conectivo, EMTC = Enfermedad mixta del tejido conectivo, EITC = Enfermedad indiferenciada del tejido conectivo, EC = Enfermedad celíaca, Anti-tTG = Anticuerpo anti-transglutaminasa $\lg \mathrm{A}, \mathrm{EMA}=$ Anticuerpo anti-endomisio. para EC, 6 pacientes tuvieron ambos anticuerpos positivos $(4,03 \%$; IC $=0,87-7,18)$ y 2 pacientes presentaron positividad sólo para tTG; dichos hallazgos se resumen en la Tabla 2. El tiempo de evolución de los pacientes seronegativos fue de 78,7 semanas y de 49,5 semanas para los seropositivos $(\mathrm{p}=0,38)$.

Los pacientes con serología positiva $(n=8)$ presentaban como diagnóstico de base LES $(\mathrm{n}=2), \operatorname{AR}(\mathrm{n}=2), \operatorname{PM}(\mathrm{n}=1), \mathrm{SS}(\mathrm{n}=1)$, APs $(n=1)$ y AI $(n=1)$; sólo la paciente con SS presentó diarrea crónica, pérdida de peso y pérdidas fetales recurrentes. Los otros pacientes no presentaban síntomas gastrointestinales. En 4 pacientes, con ambos anticuerpos positivos, se realizó diagnóstico de EC por biopsia: 1 SS, 1 PM y 2 LES. Se aplicó la clasificación de Marsh modificada, histológicamente se observó marcada atrofia en 3 casos (Marsh IIIb), en el paciente con SS y en ambos con LES. En el paciente con PM se observó atrofia parcial e hiperplasia de las criptas (Marsh IIIa). En los restantes 4 pacientes ( 2 AR, 1 APs y $1 \mathrm{AI})$ no se realizó biopsia, ya que 2 rechazaron el procedimiento y 2 se perdieron en el seguimiento.

\section{Discusión}

La EC es una enfermedad autoinmune frecuentemente subdiagnosticada; en particular en sus formas atípicas. En nuestro estudio, 5,37\% de los pacientes con diversas ETC presentó sero-

Tabla 2. Características clínicas de pacientes con anticuerpos positivos para enfermedad celíaca $(\mathbf{n}=8)$

\begin{tabular}{|ccccccccc|}
\hline $\mathbf{n}$ & Edad & Sexo & ETC & Síntomas GI & $\begin{array}{c}\text { Tiempo de evolución } \\
\text { (meses) }\end{array}$ & $\begin{array}{c}\text { Anti-tTG } \\
\text { EMA }\end{array}$ & Biopsia \\
\hline 1 & 37 & F & LES & No & 41 & + & + & + \\
2 & 42 & F & LES & No & 72 & + & + & + \\
\hline 3 & 58 & F & PM & No & 72 & + & + & + \\
4 & 49 & F & SS & Sí & 48 & + & - & NR \\
\hline 5 & 71 & M & APs & No & 23 & + & + \\
\hline 6 & 55 & F & Al & No & 24 & + & - & NR \\
7 & 34 & F & AR & No & 52 & + & + & + \\
8 & 42 & F & AR & No & 64 & + & NR \\
\hline
\end{tabular}

$\mathrm{ETC}=$ Enfermedad del tejido conectivo, EC = Enfermedad celíaca, Anti-tTG = Anticuerpo anti-transglutaminasa IgA, EMA = Anticuerpo anti-endomisio, LES = Lupus eritematoso sistémico, PM = Polimiositis, SS = Síndrome de Sjögren, APs $=$ Artritis psoriásica, $\mathrm{Al}=$ Artritis Indiferenciada, $\mathrm{AR}=$ Artritis reumatoidea, $\mathrm{NR}=$ No realizada, $\mathrm{Gl}=$ gastrointestinales. 
logía positiva para EC. Se hallaron anticuerpos positivos en pacientes con diagnóstico de SS, AR, APs, PM y LES consistente con lo reportado por otros autores.

De acuerdo a la bibliografía, el SS es la ETC que con mayor frecuencia se asocia a EC. Iltanen y col. hallaron una frecuencia de $14,7 \%$ en 34 pacientes con SS primario. En este estudio, se reconocieron como factores de susceptibilidad genética a los haplotipos HLA DQ2 y DQ8 compartidos por ambas patologías?.

En un estudio realizado en enfermedades reumatológicas autoinmunes, Koehne y col. sugieren que la positividad para antigliadina IgA e IgG y anti EMA probablemente no sea específica para detectar EC en pacientes con enfermedades reumatológicas autoinmunes ${ }^{12}$. En otro estudio, de tipo transversal, realizado por Castillo-Ortiz y col, en el cual evaluaron la presencia de anticuerpos para EC en pacientes con AR, 18,6\% presentó anticuerpos antigliadina y sólo 1 paciente anti-tTG positivos; sin embargo, no evaluaron EMA, como en nuestro protocolo ${ }^{13}$.

Comunicaciones previas demostraron la existencia de una susceptibilidad genética compartida entre diversas enfermedades autoinmunes, entre ellas: AR y EC. Ciertos polimorfismos de nucleótidos simples, tales como el TAGAP y el SH2B3 han sido asociados tanto a AR como a $\mathrm{EC}^{14-16}$.

En relación a las miositis, en nuestra población, se detectó un paciente con EC y PM. La asociación entre miopatías inflamatorias y EC ha sido documentada en niños ${ }^{17,18} \mathrm{y}$ adultos ${ }^{19}$. Otros autores, que evaluaron los títulos de autoanticuerpos para múltiples enfermedades autoinmunes en pacientes con miopatías inflamatorias; observaron que los anticuerpos para antigliadina y anti-tTG fueron más prevalentes en pacientes con miopatías inflamatorias comparados con los controles ${ }^{20}$.

En nuestro estudio, además, se realizó el diagnóstico de EC en 2 pacientes con lupus sistémico. Ludvigsson et al demostraron en un estudio poblacional prospectivo, que pacientes con EC presentan un riesgo mayor de desarrollar LES (HR: 3,49; IC $=2,48-4,90)^{21}$. Asimismo, otros han demostrado que en la coexistencia de estas enfermedades, la EC puede preceder, manifestarse de manera concomitante o posteriormente al diagnóstico de $\mathrm{LES}^{22,23}$.

En relación a otras enfermedades del tejido conectivo, la asociación entre esclerodermia y EC ha sido previamente reportada ${ }^{24}$. En el año 2009, se comunicó una prevalencia de EC de 8\% (4/50) en pacientes con ES evaluados mediante anti-tTG y EMA con confirmación anatomo-patológica ${ }^{25}$.

En nuestra serie, una paciente con artritis indiferenciada y anti-tTG y EMA positivos que podría interpretarse como posible ETC o como manifestaciones articulares asociadas a EC, negativizó dichos anticuerpos con la introducción de la dieta sin gluten; motivo por el cual la paciente no aceptó la realización de la biopsia.

De acuerdo a nuestros hallazgos, sería de utilidad realizar la evaluación serológica para EC en las ETC por la superposición de enfermedades autoinmunes entre sí; así como en artritis indiferenciadas, para determinar en quienes se debe realizar la biopsia de intestino delgado para su diagnóstico definitivo e instauración de la terapéutica adecuada. Cabe mencionar la posibilidad de falsos positivos en la detección de anticuerpos anti-tTG (tanto en población general como en pacientes con enfermedades autoinmunes) ${ }^{9}$. Las posibles razones de falsos positivos incluyen la presencia de trastornos intestinales inflamatorios crónicos (enfermedad de Crohn), enfermedades autoinmunes sistémicas, hepatopatías crónicas (hepatitis C) o enfermedades parasitarias como la giardiasis. Por otra parte, la positividad de los mismos puede representar un estadio temprano de la EC, que aún no se ha manifestado clínicamente; por lo cual en este subgrupo, se torna esencial el seguimiento con la evaluación seriada de los anticuerpos, ya que pueden representar una subpoblación con EC latente. Por último, en general, múltiples enfermedades autoinmunes (como el SS, el LES o la AR) pueden presentar hipergamaglobulinemia, en cuyo caso el método de ELISA puede presentar falsos positivos ${ }^{9}$.

Aunque no hallamos ningún paciente con estas características en nuestro estudio, como ha sido mencionado previamente, la deficiencia selectiva de IgA se asocia con enfermedad celíaca. Por ello, en los pacientes con este trastorno siempre se debe determinar la presencia de autoanticuerpos de tipo IgG. Otras potenciales debilidades o limitaciones del estudio, resultan principalmente de los aspectos metodológicos del mismo, e incluyen: el diseño descriptivo del estudio, el bajo número de pacientes incluidos y la heterogeneidad de los diagnósticos de los pacientes analizados, que podrían afectar los resultados. Debido al bajo número de 
casos analizados de algunas ETC, no podemos establecer el significado de la coexistencia entre estas patologías autoinmunes y la EC. En nuestro estudio, se realizó la confirmación histológica en 4 casos, y en los 4 restantes no se realizó por motivos personales y pérdida de seguimiento. La prevalencia general de EC por serología fue de 5,37\%. Un mayor número de pacientes sería necesario para aseverar estas observaciones.

Si bien el diagnóstico definitivo de enfermedad celíaca aún se basa en los hallazgos de la biopsia intestinal, la detección de anti-tTG IgA y/o EMA constituyen una herramienta indispensable para el diagnóstico serológico cuantitativo y la pesquisa de la enfermedad celíaca en sujetos con enfermedades del tejido conectivo y artropatías inflamatorias.

\section{Responsabilidades éticas}

Protección de personas y animales. Los autores declaran que para esta investigación no se han realizado experimentos en seres humanos ni en animales.

Confidencialidad de los datos. Los autores declaran que en este artículo no aparecen datos de pacientes.

Derecho a la privacidad y consentimiento informado. Los autores declaran que en este artículo no aparecen datos de pacientes.

\section{Conflicto de intereses}

Los autores declaran no tener ningún conflicto de intereses.

\section{Referencias}

1. Farrell RJ, Kelly CP. Celiac sprue-current concepts. N Engl J Med 2002; 346: 180-8.

2. Gómez JC, Selvaggio GS, Viola M, Pizarro B, La Motta G, de Barrio S, et al. Prevalence of celiac disease in Argentina: screening of an adult population in the La Plata area. Am J Gastroenterol 2001; 96: 2700-4.

3. Lee SK, Green PH. Celiac sprue (the great modern-day imposter). Curr Opin Rheumatol 2006; 18: 101-7.

4. Chow MA1, Lebwohl B, Reilly NR, Green PH. Immunoglobulin A deficiency in celiac disease. J Clin Gastroenterol 2012; 46 (10): 850-4.

5. James SP. National Institutes of Health Consensus Development Conference Statement on Celiac Disease, June 28-30, 2004. Gastroenterology 2005; 128: S1-9.

6. James MW, Scott BB. Coeliac disease: the cause of the various associated disorders? Eur J Gastroenterol Hepatol 2001; 13: 1119-21.

7. Iltanen S, Collin P, Korpela M, Holm K, Partanen J, Polvi A, et al. Coeliac disease and markers of coeliac disease latency in patients with primary Sjögren's syndrome. Am J Gastroenterol 1999; 94: 1042-6.

8. Lindquist U, Rudsander A, Bostrom A, Nilsson B, Michaelsson G. IgA antibodies to gliadin and coeliac disease in psoriatic arthritis. Rheumatology 2002; 41: 31-7.

9. Luft LM, Barr SG, Martin LO, Chan EK, Fritzler MJ. Autoantibodies to tissue transglutaminase in Sjögren's syndrome and related rheumatic diseases. J Rheumatol 2003; 30: 2613-9.

10. Lubrano E, Ciacci C, Ames PR, Mazzacca G, Oriente P, Scarpa R. The arthritis of coeliac disease: prevalence and pattern in 200 adult patients. Br J Rheumatol 1996; 35 : 1314-8.

11. Spadaro A, Sorgi ML, Scrivo R, Picarelli A, Tola MD, Sabbatella L, et al. Anti-tissue transglutaminase antibodies in inflammatory and degenerative arthropathies. Reumatismo 2002; 54: 344-50.

12. Koehne Vde B, Bahia M, Lanna CC, Pinto MR, Bambirra EA, Cunha AS. Prevalence of serological markers for celiac disease (IgA and IgG class antigliadin antibodies and IgA class antiendomysium antibodies) in patients with autoimmune rheumatologic diseases in Belo Horizonte, MG, Brazil. Arq Gastroenterol 2010; 47: 250-6.

13. Castillo-Ortiz JD, Durán-Barragán S, Sánchez-Ortíz A, Ramos-Remus C. Anti-transglutaminase, antigladin and ultra purified anti-gladin antibodies in patients with a diagnosis of rheumatoid arthritis. Rheumatol Clin 2011; 7:27-9.

14. Eyre S, Hinks A, Bowes J, Flynn E, Martin P, Wilson AG, et al. Overlapping genetic susceptibility variants between three autoimmune disorders: rheumatoid arthritis, type 1 diabetes and coeliac disease. Arthritis Res Ther 2010; 12 (5): R175.

15. Hinks A, Martin P, Flynn E, Eyre S, Packham J, Barton A, et al. Investigation of type 1 diabetes and celiac disease susceptibility loci for association with juvenile idiopathic arthritis. Ann Rheum Dis 2010; 69: 2169-72.

16. Zhernakova A, Stahl EA, Trynka G, Raychaudhuri S, Festen EA, Franke L, et al. Meta-analysis of genome-wide association studies in celiac disease and rheumatoid arthritis identifies fourteen non-HLA shared loci. PLoS Genet 2011; 7: e1002004.

17. Buderus S, Wagner N, Lentze MJ. Concurrence of celiac disease and juvenile dermatomyositis: Result of a specific immunogenetic susceptibility? J Pediatr Gastroenterol Nutr 1997; 25: 101-3. 
18. Falcini F, Porfirio B, Lionetti P. Juvenile dermatomyositis and celiac disease. J Rheumatol 1999; 26: 1419-20.

19. Song MS, Farber D, Bitton A, Jass J, Singer M, Karpati $G$, et al. Dermatomyositis associated with celiac disease: response to a gluten-free diet. Can J Gastroenterol Vol 2006; 20: 433-5.

20. Orbach H, Amitai N, Barzilai O, Boaz M, Ram M, Zandman-Goddard G, et al. Autoantibody screen in inflammatory myopathies high prevalence of antibodies to gliadin. Ann NY Acad Sci 2009; 1173: 174-9.

21. Ludvigsson JF, Rubio-Tapia A, Chowdhary V, Murray JA, Simard JF. Increased Risk of Systemic Lupus Erythematosus in 29,000 Patients with Biopsy-verified Celiac
Disease. J Rheumatol 2012; 39: 1964-70.

22. Zitouni M, Daoud W, Kallel M, Makni S. Systemic lupus erythematosus with celiac disease: a report of five cases. Joint Bone Spine 2004; 71: 344-6.

23. Freeman HJ. Adult celiac disease followed by onset of systemic lupus erythematosus. J Clin Gastroenterol 2008; 42: 252-5.

24. Gómez-Puerta JA, Gil V, Cervera R, Miquel R, Jiménez $\mathrm{S}$, Ramos-Casals M, et al. Coeliac disease associated with systemic sclerosis. Ann Rheum Dis 2004; 63: 104-5.

25. Rosato E, De Nitto D, Rossi C, Libanori V, Donato G, Di Tola M, et al. High incidence of celiac disease in patients with systemic sclerosis. J Rheumatol 2009; 36: 965-9.27. 